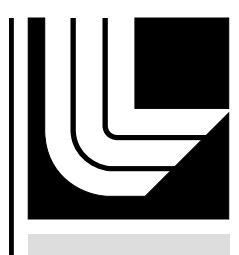

LAW RENCE LIVERMORE N A TIO N A L LABORATORY

\title{
UCRL-TR-218251
}

\section{Gamma-Ray Bursts Shower the Universe with Metals}

A. Hazi

January 18, 2006 
This document was prepared as an account of work sponsored by an agency of the United States Government. Neither the United States Government nor the University of California nor any of their employees, makes any warranty, express or implied, or assumes any legal liability or responsibility for the accuracy, completeness, or usefulness of any information, apparatus, product, or process disclosed, or represents that its use would not infringe privately owned rights. Reference herein to any specific commercial product, process, or service by trade name, trademark, manufacturer, or otherwise, does not necessarily constitute or imply its endorsement, recommendation, or favoring by the United States Government or the University of California. The views and opinions of authors expressed herein do not necessarily state or reflect those of the United States Government or the University of California, and shall not be used for advertising or product endorsement purposes.

This work was performed under the auspices of the U.S. Department of Energy by University of California, Lawrence Livermore National Laboratory under Contract W-7405-Eng-48. 


\section{Gamma-Ray Bursts Shower the Unive} with Metals

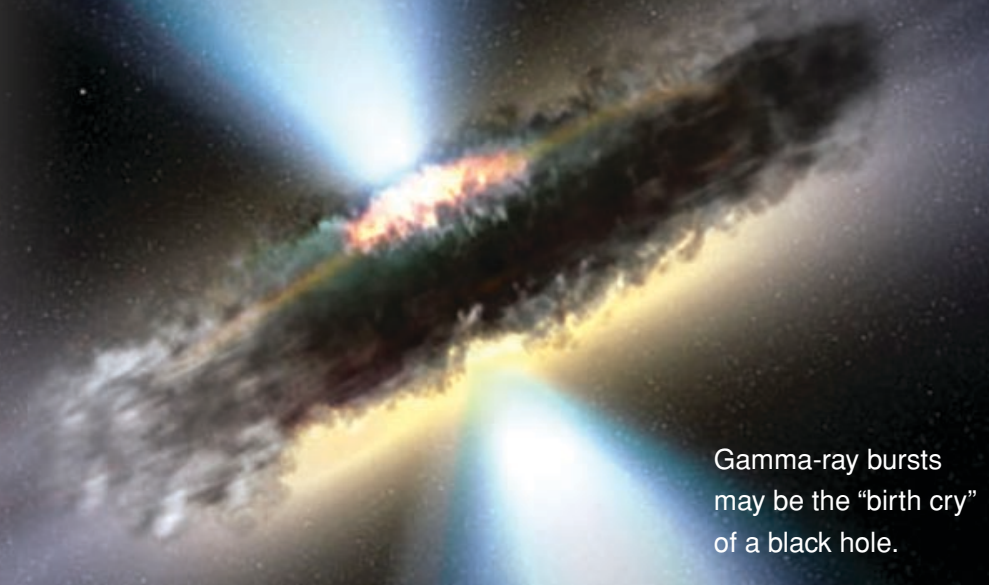

A CCORDING to the results from a Livermore computer model, some of the small change jingling in your pocket contains zinc and copper created in massive gamma-ray bursts (GRBs) that rank as the most impressive light shows in the universe.

Livermore astrophysicist Jason Pruet and his colleagues Rebecca Surman and Gail McLaughlin from North Carolina State University (NCSU) reported on their calculations in the February 20, 2004, issue of Astrophysical Journal Letters. They found that GRBs from black holes surrounded by a disk of dense, hot plasma may have contributed heavily to the galactic inventory of elements such as calcium, scandium, titanium, zinc, and copper. "A typical GRB of this kind briefly outshines all the stars in millions of galaxies combined," says Pruet. "Plus it makes about 100 times as much of some common elements as an ordinary supernova."

\section{Star Light, Star Bright}

Gamma-ray bursts were discovered during the Cold War-era research to develop space technologies for monitoring arms-control agreements. Between 1963 and 1970, the U.S. launched 12 Vela satellites to search for the $\mathrm{x}$ and gamma rays that would indicate a nuclear explosion in the upper atmosphere. These satellites detected several intense but fleeting flashes of gamma rays. The rays, however, were not from a nuclear test. They originated somewhere deep in space, but where — and what caused themremained a mystery.

"A heated debate followed," says Pruet. "Many scientists believed the bursts originated in our galaxy. If they were peppered throughout the universe, these events would be incredibly energetic." More than two decades passed before satellite technology could provide the measurement sensitivity needed to resolve this mystery. Then in 1991, when the Compton Gamma Ray Observatory (CGRO) satellite was launched, scientists began to get meaningful answers to their questions. During the satellite's 9 years on the job, it detected more than 2,600 GRBs flashing and fading in seconds or minutes, too brief to aim a telescope for follow-up observations.
The CGRO data indicated that bursts appeared from random directions in the sky. This finding led many astronomers to believe that GRBs occurred outside the Milky Way in other galaxies. Unfortunately, the CGRO satellite was not sensitive enough to pinpoint which specific galaxies hosted these spectacular explosions. Each event could only be localized to within an area large enough to contain several thousand galaxies.

The Dutch-Italian BeppoSAX X-ray astronomical satellite, which was launched in 1996, solved this sensitivity problem. By correlating X-ray data from BeppoSAX with data from groundbased observatories, astronomers could finally verify that GRBs are indeed deep in space-some more than 8 billion light years away. Thus, observed GRBs occurred in distant reaches of the universe far, far back in time.

Data from BeppoSAX proved that GRBs are not only extragalactic but also the most energetic events in the universe. If GRBs are isotropic, emitting energy in all directions equally, then the energy from some bursts would approach one solar rest massthe total energy obtained if the Sun is entirely converted to energy. Observations in the last few years indicate that GRBs are not isotropic but, instead, are beamed to about 1 percent of the sky. The total energy emitted is still enormous, but more plausible.

The BeppoSAX data have helped scientists understand the energy of GRBs. Their next challenge was to determine what causes the powerful bursts. "Several hundred papers appeared advancing different ideas," says Pruet. "For example, some proposed that GRBs are caused by 'puffy' neutron stars or evaporating black holes. Other researchers suggested the GRBs are supermassive stars that are the grandparents of today's stars."

Today, the leading model for explaining these mysterious events is the collapsar, or collapsing star, model proposed by Stan Woosley, a professor of astronomy and astrophysics at the University of California at Santa Cruz (UCSC) and a longtime Livermore collaborator. According to the collapsar model, a GRB arises when a dying rotating star is too massive to successfully explode as a supernova. Instead, the star collapses into a black hole

\section{Reprinted from Science \& Technology Review, March 2005}

UCRL-TR-218251 
surrounded by a dense accretion disk, which spirals into the black hole like water flowing down a drain. The GRB - the "birth cry" of the black hole-is produced by gravitational energy liberated as disk material falls into the black hole.

\section{Pennies from Heaven}

Another interesting twist to the mystery emerged a few years ago. Data show that a fraction of GRBs emits light with characteristics similar to emissions from ordinary supernovae. The supernovalike light curves indicate that these extremely energetic explosions are spewing out about one-half of a solar mass of radioactive nickel at thousands of kilometers per second. "A couple of theories have been proposed to explain how this nickel is created," says Pruet.

One proposal is that the nickel is created from "explosive burning," much like what happens in ordinary supernovae. In explosive burning, a violent shock traversing the stellar mantle heats nuclei and rips them apart. As the nucleons cool, they recombine to form radioactive nickel and progenitors of other common elements. "However," says Pruet, "simulations of exploding stars show that nickel production through explosive burning in a collapsar is unlikely."

Another possibility, first suggested by Woosley and his colleague Andrew MacFadyen, is that the nickel originates from a wind blown off the accretion disk. "The cold matter making up the star's inner layers is drawn into the disk by the enormous pull of the black hole," Pruet says. "As the matter moves closer to the black hole, it becomes hotter and denser, dissociating into its nuclear components-neutrons and protons. One might guess that this disk material is doomed; however, about half of the accretion disk escapes in a viscous wind before it is consumed by the black hole. New nuclei, including iron, are synthesized as this wind cools and the radioactive nickel decays."

Pruet and his colleagues are also investigating whether collapsars might be the sources of other elements as well. Livermore's work on this project is funded through a grant Woosley received from the Department of Energy's (DOE's) Program for Scientific Discovery through Advanced Computing. The NCSU team received DOE funding by winning an Outstanding Junior Investigator Award.

"Rather than develop a theory describing the disk wind, we began with the assumption that a wind is responsible for the observed abundance of nickel," says Pruet. "Just using this simple assumption allows us to calculate the nucleosynthesis of other elements in the wind." The team then modeled the outflow and used large nuclear reaction network calculations to examine diskwind nucleosynthesis. These reaction codes, maintained by Livermore scientist Rob Hoffman, describe the "burning" of nuclei from neutrons and protons to much heavier elements.

The researchers found that GRBs also produce smaller but still enormous quantities of such everyday elements as zinc, titanium,

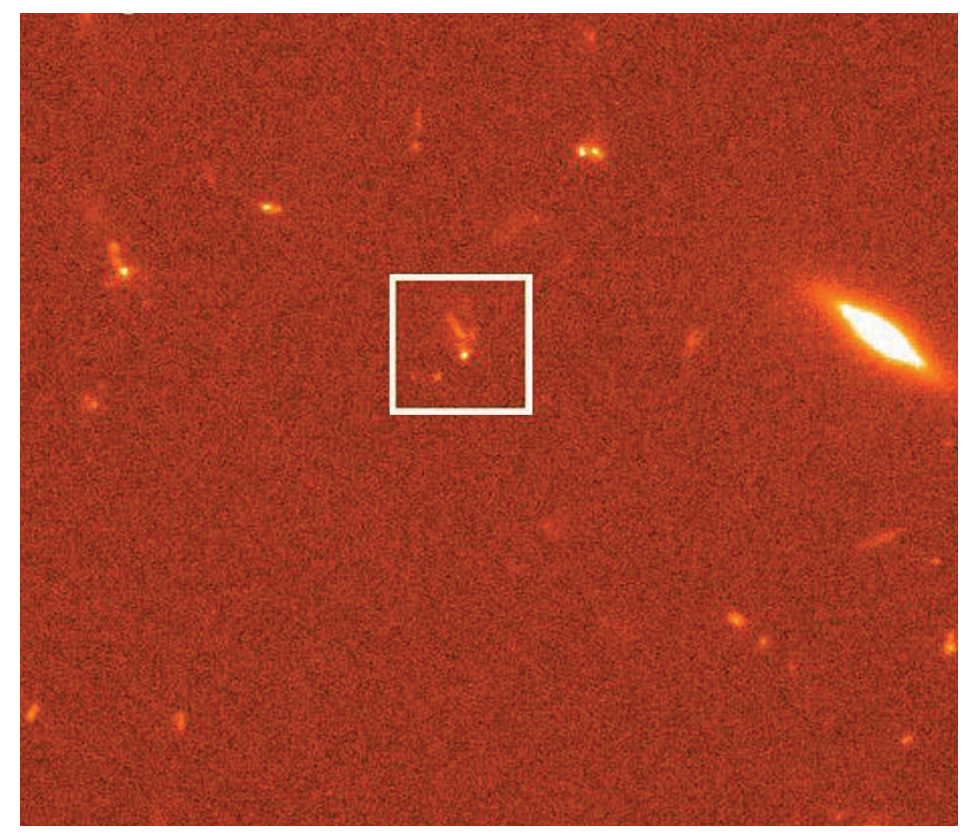

Gamma-ray bursts, such as the one in the box, not only outshine all the stars in their own galaxies but also contribute to the galactic inventory of many common elements.

copper, and scandium. Only a few stars in every hundred are expected to produce GRBs when they die. However, according to the team's calculations, these rare events probably account for as much of some elements as do all other stellar explosions combined. To put this finding in perspective, says Pruet, consider that a modern penny is 97.5 percent zinc and 2.5 percent copper. Then the raw material for about 20 pennies in every dollar may have narrowly escaped being eaten by a black hole.

\section{More Answers on the Horizon}

This research may help explain other curious phenomena as well. For example, some observations indicate that x-ray emission lines may be associated with collapsars, and some scientists speculate that those lines come from iron. Others have suggested that the emission lines are from nickel, perhaps being ejected at high velocity behind a jet emitted from the poles of the collapsar. Both the existence and source of these lines are debated.

"If a disk wind is producing radioactive nickel," says Pruet, "then its decay product, iron, is by far the most abundantly produced element in the wind. The second most abundant element-generally zinc_-is much less, 5 percent or less of the total elements produced." If the radioactive nickel, which lives for about a week, is responsible for the x-ray emission lines, then scientists should find an initial strong line or lines from nickel with much weaker lines from other elements such as zinc. 
Further research on GRBs may also help scientists understand the history of element production in the Milky Way galaxy by providing a record of events, much like fossils provide a record of geologic events on Earth. "If these bursts make sizable contributions to the inventory of scandium, titanium, copper, and

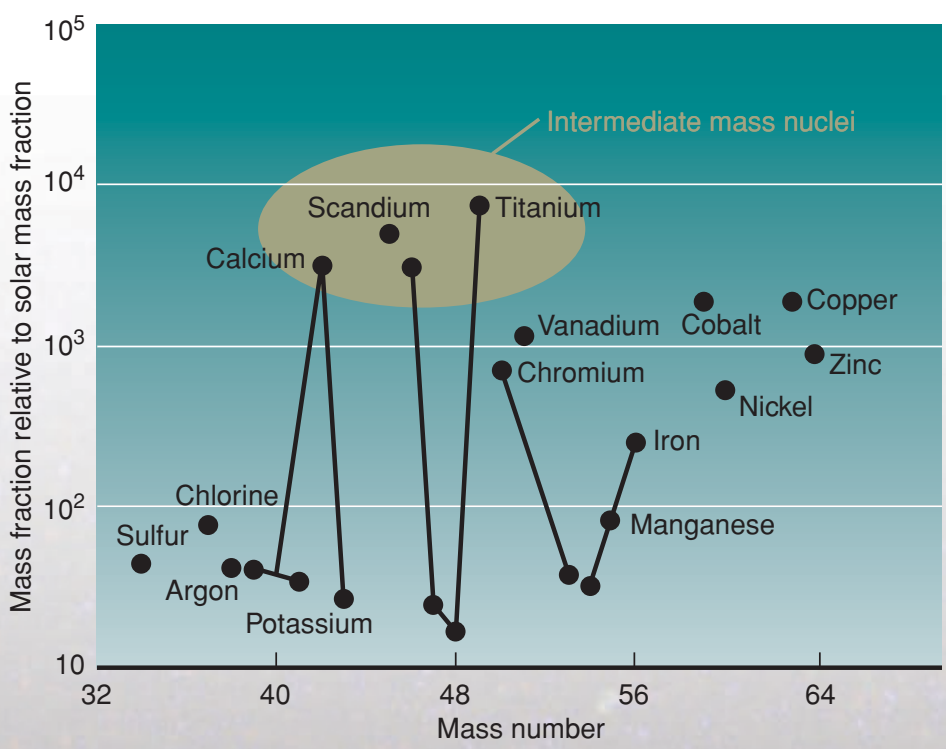

Calculations for nucleosynthesis in a collapsar wind indicated that collapsars and their attendant gamma-ray bursts could be the source of elements such as scandium, titanium, zinc, and copper. zinc in the universe, we should be able to read the history of GRBs in the nuclear abundance patterns of stars," says Pruet. "There's likely a signature of past GRBs in stars. For instance, as a star explodes, the mass is mixed in the galaxy. When the mass collapses inward, another star is born. Thus, if we find a star showing a lot of scandium, we might be able to say that, in the past, a GRB occurred near this star's birthplace."

By examining material expelled from black holes near GRBs, scientists may also gain insights into where the heavy elementsgold, silver, plutonium, and uranium-were first formed.

Synthesizing these elements requires neutron-rich conditions, and if the team's calculations are right, the inner area of black-hole accretion disks provides such a source. The answer to this question and others may indeed be blowing in the wind.

-Ann Parker

Key Words: accretion disk, black hole, collapsar model, copper, disk wind, gamma-ray burst (GRB), neutron star, nickel, nuclear reaction network, nucleosynthesis, scandium, supernova, zinc.

\section{For further information contact Jason Pruet (925) 422-5850 (pruet1@IInl.gov).}

